Research Article

\title{
Auraptene, a Monoterpene Coumarin, Inhibits LTA-Induced Inflammatory Mediators via Modulating NF- $\kappa$ B/MAPKs Signaling Pathways
}

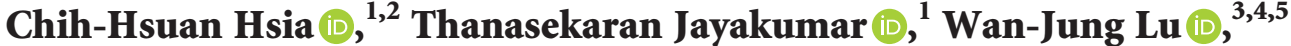 \\ Joen-Rong Sheu $₫{ }^{1}{ }^{1}$ Chih-Wei Hsia $\left(\mathbb{1},{ }^{1}\right.$ Periyakali Saravana Bhavan $\left(\mathbb{1},{ }^{6}\right.$ \\ Manjunath Manubolu $\left(\mathbb{0},{ }^{7}\right.$ Wei-Chieh Huang $\mathbb{C}^{1},{ }^{1}$ and Yi Chang $\mathbb{C}^{8,9}$ \\ ${ }^{1}$ Graduate Institute of Medical Sciences, College of Medicine, Taipei Medical University, Taipei 110, Taiwan \\ ${ }^{2}$ Translational Medicine Center, Shin Kong Wu Ho-Su Memorial Hospital, Taipei 111, Taiwan \\ ${ }^{3}$ Department of Pharmacology, School of Medicine, College of Medicine, Taipei Medical University, Taipei 110, Taiwan \\ ${ }^{4}$ Department of Medical Research, Taipei Medical University Hospital, Taipei 110, Taiwan \\ ${ }^{5}$ Graduate Institute of Metabolism and Obesity Sciences, College of Nutrition, Taipei Medical University, Taipei 110, Taiwan \\ ${ }^{6}$ Department of Zoology, Bharathiar University, Coimbatore 641046, Tamil Nadu, India \\ ${ }^{7}$ Department of Evolution, Ecology and Organismal Biology, Ohio State University, Columbus, OH 43212, USA \\ ${ }^{8}$ School of Medicine, Fu Jen Catholic University, New Taipei City 242, Taiwan \\ ${ }^{9}$ Department of Anesthesiology, Shin Kong Wu Ho-Su Memorial Hospital, Taipei 111, Taiwan
}

Correspondence should be addressed to Yi Chang; m004003@ms.skh.org.tw

Received 10 June 2021; Accepted 1 November 2021; Published 16 November 2021

Academic Editor: Weicheng $\mathrm{Hu}$

Copyright (c) 2021 Chih-Hsuan Hsia et al. This is an open access article distributed under the Creative Commons Attribution License, which permits unrestricted use, distribution, and reproduction in any medium, provided the original work is properly cited.

\begin{abstract}
Objective. Oxidative stress-mediated inflammatory events involve in the progress of several diseases such as asthma, cancers, and multiple sclerosis. Auraptene (AU), a natural prenyloxycoumarin, possesses numerous pharmacological activities. Here, the antiinflammatory effects of AU were investigated in lipoteichoic acid- (LTA-) induced macrophage cells (RAW 264.7). Methods. The expression of cyclooxygenase (COX-2), tumor necrosis factor (TNF- $\alpha$ ), interleukin-1 $\beta$ (IL-1 $\beta$ ), and inducible nitric oxide synthase (iNOS) and the phosphorylation of extracellular signal-regulated kinase (ERK) 1/2, p38 MAPK, c-Jun N-terminal kinase (JNK), heme oxygenase (HO-1), p65, and $\mathrm{I} \kappa \mathrm{B} \alpha$ were all identified by western blotting assay. The level of nitric oxide (NO) was measured by spectrometer analysis. The nuclear translocation of p65 nuclear factor kappa B (NF- $\kappa \mathrm{B})$ was assessed by the confocal microscopic staining method. Native polyacrylamide gel electrophoresis was performed to perceive the activity of antioxidant enzyme catalase (CAT). Results. AU expressively reduced NO production and COX-2, TNF- $\alpha$, IL- $1 \beta$, and iNOS expression in LTA-stimulated cells. AU at higher concentration $(10 \mu \mathrm{M})$ inhibited ERK and JNK, but not p38 phosphorylation induced by LTA. Moreover, AU blocked $\mathrm{I} \kappa \mathrm{B}$ and $\mathrm{p} 65$ phosphorylation, and $\mathrm{p} 65$ nuclear translocation. However, AU pretreatment was not effective on antioxidant HO-1 expression, CAT activity, and reduced glutathione (GSH, a nonenzymatic antioxidant), in LTA-induced RAW 264.7 cells. Conclusion. The findings of this study advocate that AU shows anti-inflammatory effects via reducing NF- $\kappa$ B/ MAPKs signaling pathways.
\end{abstract}

\section{Introduction}

Various chemicals and pathogens considered as harmful stimuli produce inflammation, which is a protective response of our body. Inflammation can be classified as acute and chronic, which induces pain and tissue injuries. Rapid onset and short duration of action can be noticed in the acute form, which is facilitated by the excretion of numerous cytokines including interleukin-1 (IL-1), IL-6, IL-11, IL-8, and tumor necrosis factor-alpha (TNF- $\alpha$ ) 
$[1,2]$. Nevertheless, in chronic inflammation, persistence of the inflammatory reactions could induce the migration of lymphocytes and macrophages to the damaged tissues [3]. Chronic inflammatory responses have been associated with the progression of various diseases such as asthma, arthritis, and neurodegenerative disorders [4]. Studies have established the involvement of several mediators including prostaglandin E2 (PGE2) in inflammatory events. Various symptoms including bone metabolism, wound healing, kidney function, blood vessel, and the immune responses have been associated with PGE2 secretion [5]. Cyclooxygenase (COX-2) protein can be expressed in response to physical, chemical, and biological stimulation [6]. The production of PGE2 can be augmented by COX-2, which denotes a central step in the events of inflammation.

Oxidative stress is known to be induced by elevated reactive oxygen species (ROS) and nitric oxide (NO) or reduced antioxidant enzymes catalase (CAT) and superoxide dismutase (SOD) and nonenzymatic glutathione (GSH) $[7,8]$. Studies have indicated that oxidative stress plays a major role in the progress of inflammatory diseases [9]. The major component of Gram-positive bacteria, lipoteichoic acid (LTA), induces pathogenesis of sepsis [10] and lung injury by producing inflammatory reactions [11]. Therefore, examining the mechanisms that control LTAstimulated cell activation is important for the analysis and treatment of lung inflammatory diseases. This bacterial component stimulates the release of IL- $1 \beta$, IL- 6 , and TNF- $\alpha$ [12]. LTA induces TNF- $\alpha$ and IL- 6 expressions by inducing the phosphorylation of ERK1/2 in macrophages, and it also activates nuclear translocation of nuclear factor- (NF-) $\kappa \mathrm{B}$ from the cytoplasm [13]. It has been proposed that various plant-based natural components have reported to have antiinflammatory effects through suppressing inflammationassociated mediators and enhancing antioxidant defense molecules.

Auraptene, a geranyloxyl moiety of C-7 (7-geranyloxycoumarin), is a promising and most rich natural prenyloxycoumarin compound [14]. Plants of the Rutaceae family are the highest source of auraptene, and it is also the most general component of citrus fruits. Hence, citrus species are the major natural source of auraptene [14]. Several exciting pharmacological activities have been reported for this bioactive phytochemical such as antioxidant [15], anti-inflammatory [16], antimicrobial [17], antigenotoxic [18], neuroprotective [19], and immunomodulatory properties [20]. Murakami et al. [16] had well discussed the effect of auraptene in inflammation-mediated carcinogenesis. A study specified that dietary supplementation of auraptene in mice diminishes pulmonary metastasis of B16BL6 melanoma cells and prevents the growth of metastatic tumors in the lungs via inducing apoptosis [21]. In addition, auraptene showed promising effects of wound healing through inhibiting the secretion of inflammatory mediators in vitro, including IL-6 and IL8 [22]. Hence, this study aimed to assess the anti-inflammatory mechanism of auraptene against LTA-stimulation in RAW 264.7 cells.

\section{Materials and Methods}

2.1. Materials. RAW 264.7 cells were obtained from the American Type Culture Collection (ATCC, Manassas, VA, USA, TIB-71). Auraptene (AU, $>98 \%$, Figure 1(a)) was purchased from ChemFaces Biochem, Wuhan, Hubei, China. Sigma (St Louis, MO, USA) supplied potassium ferricyanide, ferric chloride, and dimethyl sulfoxide (DMSO). Santa Cruz Biotechnology (Dallas, TX, USA) supplied anti-iNOS and COX-2 polyclonal antibodies (pAb). We purchased antibodies against TNF- $\alpha$, phospho-p38 MAPK Thr180/Tyr182, phospho-c-JNK (Thr183/Tyr185), phospho-p44/p42 ERK (Thr202/Tyr204), phospho-I $\kappa \mathrm{B} \alpha$ Ser32/36, and phospho-NF- $\kappa$ B p65 (Ser536) pAbs from Cell Signaling (Beverly, MA, USA). Anti-IL-1 $\beta$ and anti-HO-1 pAbs were purchased from BioVision (Milpitas, CA, USA) and Enzo (Farmingdale, New York, USA), respectively. The antibody against $\alpha$-tubulin was purchased from NeoMarkers (Fremont, CA, USA). AU was dissolved in 0.1\% DMSO.

2.2. Cell Viability and Morphology of RAW Cells. RAW 264.7 cells were cultivated in Dulbecco's Modified Eagle's Medium $(\mathrm{DMEM})$ at $37^{\circ} \mathrm{C}$ under $5 \% \mathrm{CO}_{2}$ and $95 \%$ air. At a concentration of $1 \times 10^{5}$ cells/well, they were pretreated with $\mathrm{AU}$ (5-20 $\mu \mathrm{M})$ for $24 \mathrm{~h}$. The 3-(4,5-dimethylthiazol-2-yl)-2,5diphenyl-2H-tetrazolium bromide (MTT) assay was used to measure cell viability in which $5 \mathrm{mg} / \mathrm{mL}$ of MTT working solution was added to the culture medium. The formation of crystals was digested by suing $300 \mu \mathrm{l}$ of DMSO. The formula of absorbance of treated cells/absorbance of control cells $\times 100 \%$ is used to measure the cell viability index.

2.3. Measurement of NO Production. To estimate the level of $\mathrm{NO}, \mathrm{AU}$ at 5 and $10 \mu \mathrm{M}$ was added to cells with or without LTA $(5 \mu \mathrm{g} / \mathrm{ml})$ for $24 \mathrm{~h}$ in the medium. Briefly, a $100 \mu \mathrm{l}$ equal volume of culture suspension and Griess reagent was mixed and incubated for $10 \mathrm{~min}$. NO levels were estimated by quantifying nitrite levels by an MRX absorbance reader with the optical density at $550 \mathrm{~nm}$.

2.4. Immunoblotting Assay. The equal amount $(50 \mu \mathrm{g})$ of proteins from $6 \times 10^{5}$ cells were run on $12 \%$ sodium dodecyl sulphate-polyacrylamide gel electrophoresis (SDS-PAGE) gels. The separated proteins were transferred to polyvinylidene difluoride (PVDF) membranes and then blocked using 5\% skim milk for 40 min. After blocking, the membrane was titrated with different primary antibodies of targeted proteins for $2 \mathrm{~h}$ and consequently incubated with anti-rabbit IgG or sheep anti-mouse IgG for $1 \mathrm{~h}$. The intensity of protein bands was measured by using the Biolight Windows Application, V2000.01 (Bio-Profil, Vilber Lourmat, France) software.

2.5. Confocal Microscopy Assay. Cells were seeded at $5 \times 10^{4} /$ well, cultured on cover slips, and treated by AU $(10 \mu \mathrm{M})$ for $30 \mathrm{~min}$ and then triggered by LTA $(5 \mu \mathrm{g} / \mathrm{ml})$ for $1 \mathrm{~h}$. Coverslips were successively fixed with $4 \%$ 
<smiles>CC(C)=CCC/C(C)=C/COc1ccc2ccc(=O)oc2c1</smiles>
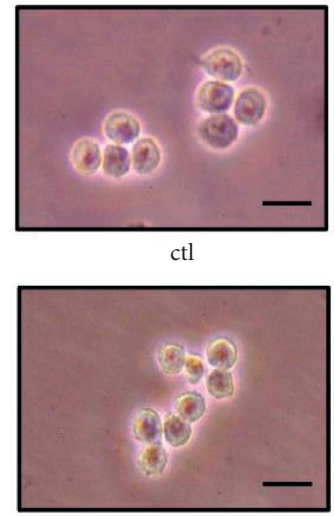

(a)

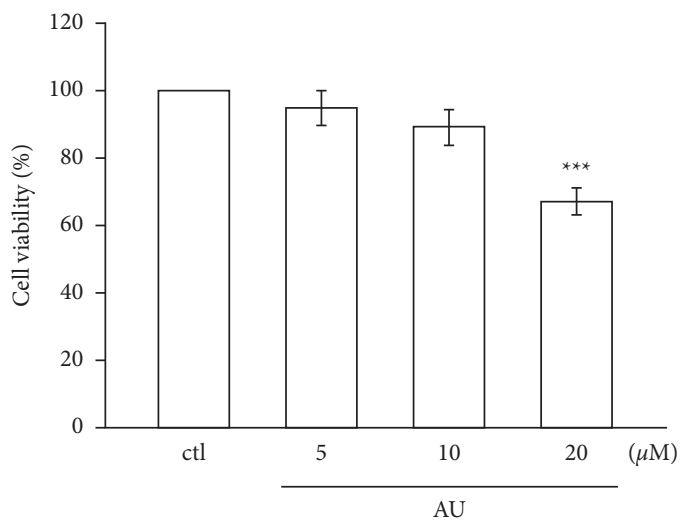

(c) ctl

$10 \mu \mathrm{M} \mathrm{AU}$

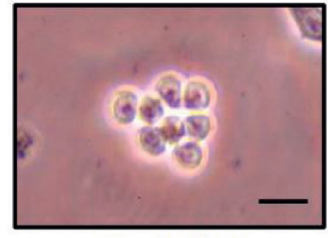

$5 \mu \mathrm{M} \mathrm{AU}$

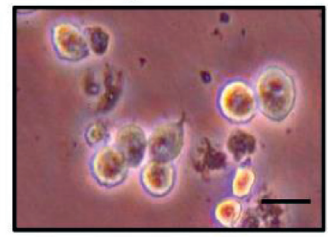

$20 \mu \mathrm{M} \mathrm{AU}$

(b)

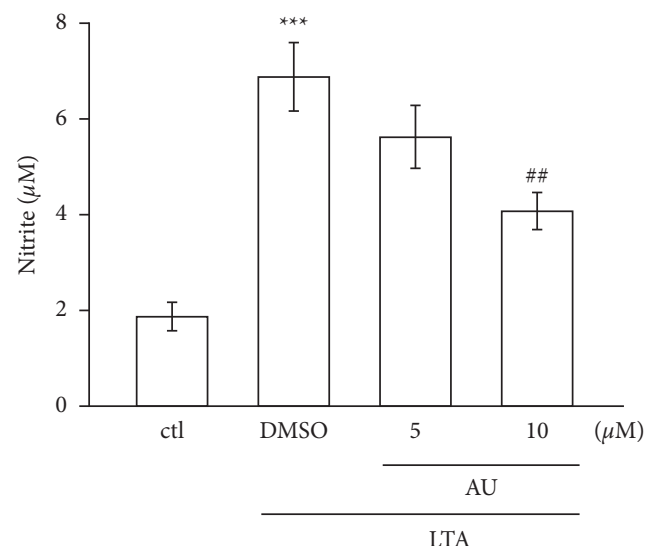

(d)
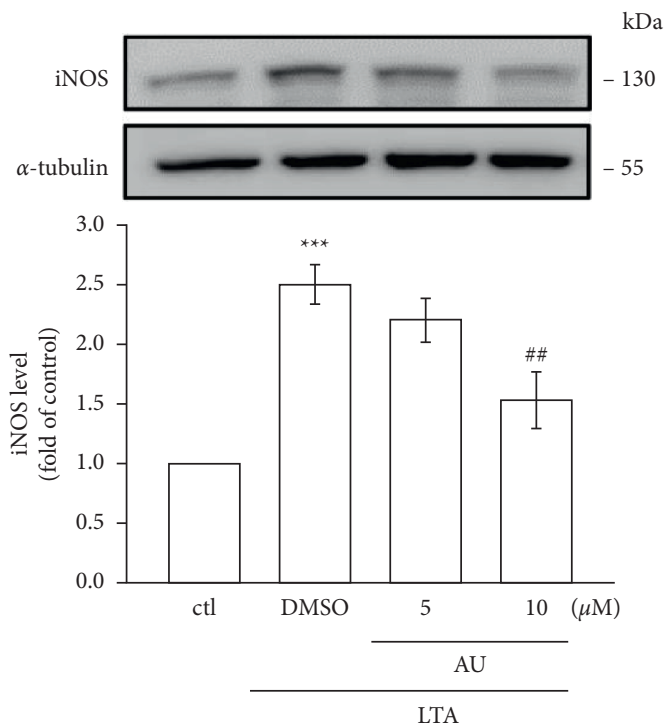

(e)

FIgURE 1: Chemical structure of auraptene (AU) and the effects of AU on morphology and cell viability and on LTA-induced NO production and iNOS expression in RAW 264.7 cells. (a) Chemical structure of AU. (b), (c) Cells were pretreated with AU (5, 10, or 20 $\mu$ M) for 24. Cell viabilities were determined by the MTT assay. Scale bar $=25 \mu \mathrm{m}$. (d), (e) Cells were untreated or pretreated with AU (5 and $10 \mu \mathrm{M})$ for $30 \mathrm{~min}$ prior to stimulation with LTA $(5 \mu \mathrm{g} / \mathrm{ml})$ for $24 \mathrm{~h}$. Control cells were not treated with LTA or AU. NO was measured using the Griess reaction assay. iNOS expression was detected using western blotting assay. The values shown are the means \pm S.E.M. of four independent experiments. ${ }^{* * *} P<0.001$ vs. the control cells; ${ }^{\# \#} P<0.01$ vs. LTA-stimulated cells. 
paraformaldehyde for $10 \mathrm{~min}$ at $37^{\circ} \mathrm{C}$, double washed using PBS, incubated with $0.1 \%$ Triton X-100 for $10 \mathrm{~min}$, and then, blocked with 5\% BSA for $1 \mathrm{~h}$. Besides, the primary p65 antibody was added over the coverslips at $4^{\circ} \mathrm{C}$ overnight, and then, secondary goat anti-rabbit IgG antibody was incubated for $1 \mathrm{~h}$ at $37^{\circ} \mathrm{C}$. 4,6-Diamidino-2 phenylindole (DAPI) was used to stain nuclei in cells. The location of nuclear translocation of p65 was spotted by using the Leica TCS SP5 confocal spectral microscope imaging system (Mannheim, Germany).

2.6. Detection of Antioxidant Enzyme Catalase (CAT). According to the method defined by Woodbury et al. [23], a native polyacrylamide gel electrophoresis (NATIVE-PAGE) was run to spot the relative banding patterns of antioxidant enzyme catalase (CAT). To this analysis, unlike normal SDSPAGE, the running buffers and protein samples did not heat and omit SDS. The equal amounts of $50 \mu \mathrm{g}$ proteins were run in $8 \%$ PAGE.

2.7. Statistical Analysis. The results are presented as mean \pm standard error (S. E. M). The statistical difference among the groups was determined using one-way analysis of variance (ANOVA). Statistical alterations were detected significant. The $P$ value of the Student-Newman-Keuls test was regarded as $P<0.05$.

\section{Results}

3.1. AU Did Not Affect the Viability and Morphology of RAW 264.7 Cells. Cell morphology and viability were studied to evaluate the toxic effect of AU in RAW 264.7 cells. Among the tested concentrations of 5,10 , and $20 \mu \mathrm{M}$ AU in RAW cells for $24 \mathrm{~h}, 5$ and $10 \mu \mathrm{M}$ did not affect cell morphology as well as viability (Figures 1(b) and 1(c)), respectively. However, AU at $20 \mu \mathrm{M}$ significantly affected the morphology and viability of RAW cells. Thus, AU at feasible concentrations of 5 and $10 \mu \mathrm{M}$ were used for the subsequent investigation.

\subsection{LTA-Induced NO Production and iNOS Were Inhibited by} $A U$. Griess reaction was applied to measure the level of NO production in AU pretreated LTA-induced RAW 264.7 cells. Systemic inflammatory events have been reported to induce a proinflammatory mediator NO [24]. A rate-limiting enzyme, inducible nitric oxide synthase (iNOS), regulates the production of NO [25]. To examine if AU inhibits NO production via the modulation of iNOS expression, the expression of iNOS was detected as shown in Figure 1(e). Figures 1(d) and 1(e) show that, at a high concentration of $10 \mu \mathrm{M}$, AU significantly inhibited the LTA-induced production of $\mathrm{NO}$ and its enzyme iNOS expression (control: $1 \pm 0$, DMSO: $2.5 \pm 0.2,5 \mu \mathrm{M}: 2.2 \pm 0.2,10 \mu \mathrm{M}: 1.5 \pm 0.2)$ in RAW 264.7 cells. This result apprehends that the inhibition of iNOS expression by AU may be involved in the inhibition of LTA-induced NO production.
3.3. AU Inhibited LTA-Induced IL-1 $\beta, T N F-\alpha$, and COX-2 Expressions. LTA stimulated the levels of COX-2 (2.1 \pm 0.3 , $P<0.01), \mathrm{IL}-1 \beta(3.1 \pm 0.3, P<0.001)$, and TNF- $\alpha(3.3 \pm 0.4$, $P<0.001)$ dramatically compared to the nonstimulated control RAW cells (Figures 2(a)-2(d)). In contrast, AU at 5 and $10 \mu \mathrm{M}$ distinctly alleviated COX-2 (5 $\mu \mathrm{M}: 1.5 \pm 0.2$, $10 \mu \mathrm{M}: 1.1 \pm 0.2)$, IL $-1 \beta(5 \mu \mathrm{M}: 1.9 \pm 0.3,10 \mu \mathrm{M}: 0.7 \pm 0.1)$, and TNF- $\alpha(5 \mu \mathrm{M}: 1.7 \pm 0.3,10 \mu \mathrm{M}: 0.9 \pm 0.2)$ induced by LTA. Moreover, AU more prominently inhibited IL- $1 \beta$ and TNF- $\alpha$ (Figures 2(c) and 2(d)).

3.4. AU Inhibits ERK1/2 and JNK1/2, But Not p38 MAPK Phosphorylation. We examined the effect of AU on LTAinduced mitogen-activated protein kinases (MAPKs), since several studies have shown that these molecules actively involve on inflammation-related events. Figure 3 shows the elevated phosphorylation of ERK1/2 (3.1 \pm 0.5$)$, JNK1/2 $(3.2 \pm 0.3)$, and p38 MAPK $(3.2 \pm 0.3)$ in LTA-induced RAW cells compared to control cells. However, AU at a higher concentration of $10 \mu \mathrm{M}$ significantly diminished the LTAinduced phosphorylation of JNK1/2 (1.9 \pm 0.2$)$, and it concentration-dependently inhibited the ERK1/2 phosphorylation ( $5 \mu \mathrm{M}: 1.8 \pm 0.4,10 \mu \mathrm{M}: 1.4 \pm 0.2)$; however, it is not effective on p38 ( $5 \mu \mathrm{M}: 2.9 \pm 0.4,10 \mu \mathrm{M}: 2.8 \pm 0.2)$. These outcomes designated that AU reveals its inhibitory effects in LTA-induced inflammatory events in RAW 264.7 cells via suppressing ERK1/2 and JNK1/2 signaling cascade.

3.5. LTA-Induced NF- $\kappa B$ Signaling Pathway Was Inhibited by $A U$. NF- $\kappa \mathrm{B}$, a major transcription factor, is constantly inducing proinflammatory mediators and cytokines. This transcription factor translocates to the nucleus once it activates and binds with target DNA and then controls the activation of numerous inflammatory cytokines [25]. Here, the inhibitory effect of AU on NF- $\kappa \mathrm{B}$ signaling pathways was examined by investigating the phosphorylations of $\mathrm{I} \kappa \mathrm{B} \alpha$ and p65 and also the nuclear translocation of p65 in LTA-induced RAW cells. The results showed that AU reduced LTAinduced $\mathrm{I} \kappa \mathrm{B} \alpha$ (DMSO: $4.0 \pm 0.7,5 \mu \mathrm{M}: 2.5 \pm 0.5$, and $10 \mu \mathrm{M}$ : $1.4 \pm 0.3$ ) and p65 phosphorylation (DMSO: $3.5 \pm 0.3,5 \mu \mathrm{M}$ : $2.7 \pm 0.2$, and $10 \mu \mathrm{M}: 1.9 \pm 0.2$ ) (Figures $4(\mathrm{a})$ and $4(\mathrm{~b})$ ) and withdrew the nuclear translocation of p65 (Figure 4(c)). These results demonstrate that AU's anti-inflammatory effect in LTA-induced cells may probably be via inhibiting the $\mathrm{NF}-\kappa \mathrm{B}$ signaling pathway.

\subsection{AU Enhances Antioxidant Defense Molecules.} Oxidative stress occurs by the elevated levels of reactive oxygen species (ROS) and NO or reduced levels of antioxidant defense molecules, such as reduced glutathione (GSH), catalase (CAT), and superoxide dismutase (SOD) [7]. Numerous studies have established that oxidative stress could induce the progress of inflammatory diseases [26]. LTA stimulation in RAW cells has been demonstrated to decrease in the expression of HO-1 $(1.7 \pm 0.3)$, antioxidant enzyme catalase, and the nonenzymatic GSH (Figures 5(a)$5(\mathrm{c}))$. AU pretreatment was not effective on LTA-stimulated 


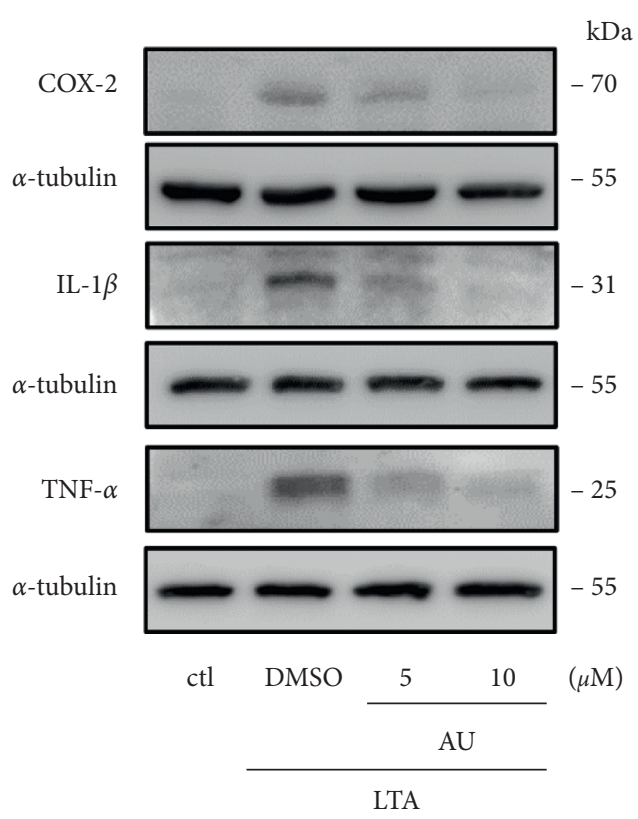

(a)

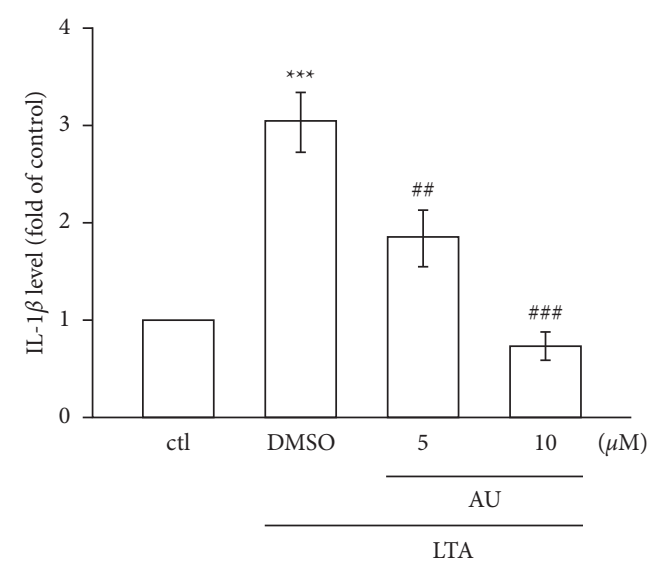

(c)

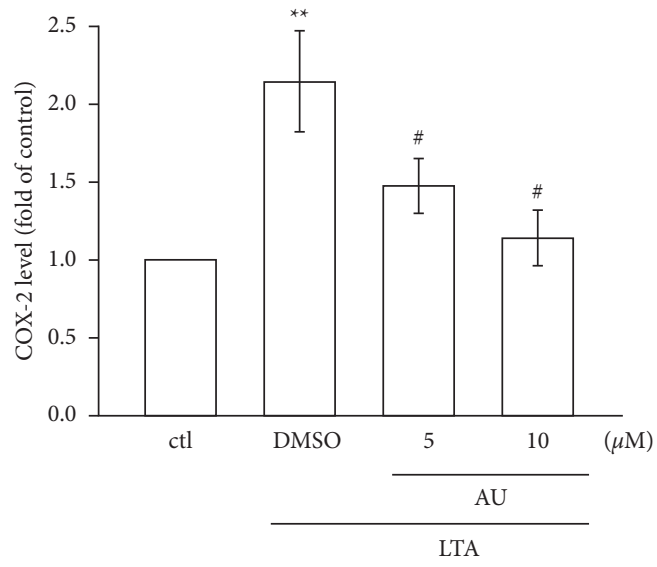

(b)

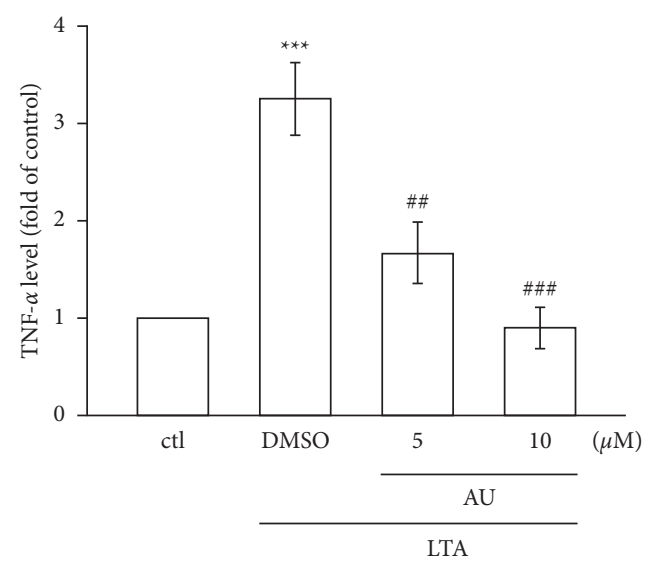

(d)

FIGURE 2: Effects of AU on the LTA-induced expression COX-2, IL-1 $\beta$, and TNF- $\alpha$ in RAW 264.7 macrophages. (a)-(d) Cells were untreated or pretreated with AU ( 5 and $10 \mu \mathrm{M})$ for $30 \mathrm{~min}$ and then stimulated with LTA $(5 \mu \mathrm{g} / \mathrm{ml})$ for $24 \mathrm{~h}$. COX-2, IL- $1 \beta$, and TNF- $\alpha$ were detected as described in Section 2. The values shown are the means \pm S.E.M. of four independent experiments. ${ }^{* *} P<0.01$ and ${ }^{* * *} P<0.001$ vs. the control cells; ${ }^{\#} P<0.05,{ }^{\# \#} P<0.01$, and ${ }^{\# \# \# ~} P<0.001$ vs. LTA-stimulated cells.

reduction of HO-1 $(5 \mu \mathrm{M}: 2.4 \pm 0.4,10 \mu \mathrm{M}: 2.3 \pm 0.3)$, CAT, and GSH in RAW cells. These results indicate that the antioxidant defense systems could not play a role in AUmediated anti-inflammatory effects in LTA-stimulated RAW cells.

\section{Discussion}

Auraptene (AU), a natural prenyloxycoumarin, is mostly present in citrus fruits. Auraptene (AU) possesses numerous pharmacological properties such as anticancer, antibacterial, antioxidant, and antiinflammatory [27]. Here, we found that auraptene ( 5 and $10 \mu \mathrm{M}$ ) did not display cytotoxicity in both control and LTA-stimulated RAW cells. Hence, the ideal concentrations of 5 and $10 \mu \mathrm{M}$ of auraptene were used in this study. A study exposed that auraptene at concentrations of 5-40 $\mu \mathrm{M}$ had no cytotoxicity on murine lymphocytes [28]. Together, as revealed in the present study, anti-inflammatory and antioxidative effects of auraptene are not through its cytotoxicity. Moreover, this study found that anti-inflammatory effects of $\mathrm{AU}$ was facilitated via preventing the production of NO and its enzyme iNOS expression. Auraptene also inhibited the LTA-induced protein expression of IL- $1 \beta$ and TNF- $\alpha$ by inhibiting the mitogen activated protein kinases (MAPKs)/NF- $\kappa \mathrm{B}$ pathways.

As it is established, proinflammatory cytokines and mediators such as NO, IL- $1 \beta$, IL- 6 , and TNF- $\alpha$ play a major role in the inflammatory process. Chronic inflammation has been reported to cause several diseases such as cancers, arthritis, and cardiovascular diseases [29]. A recent study 


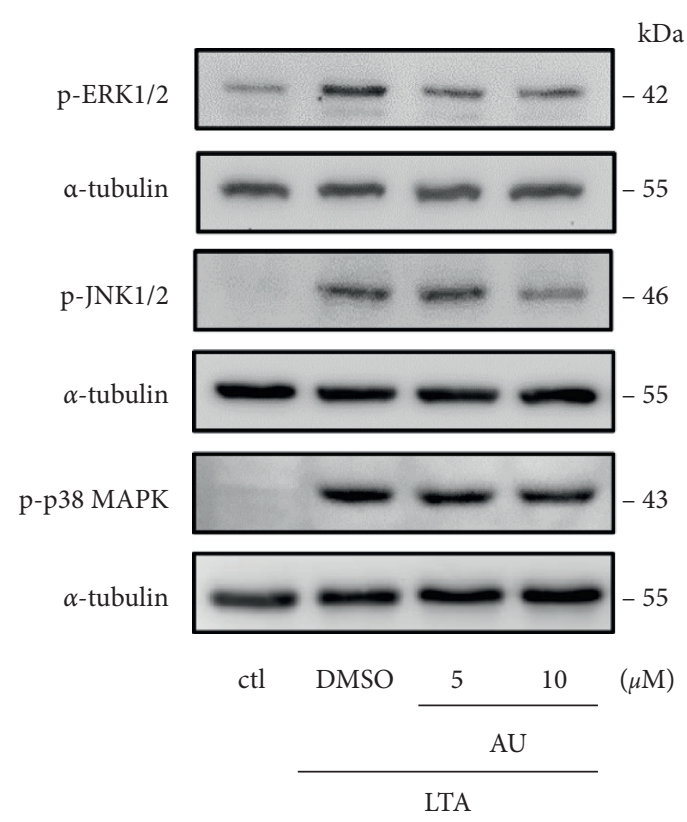

(a)

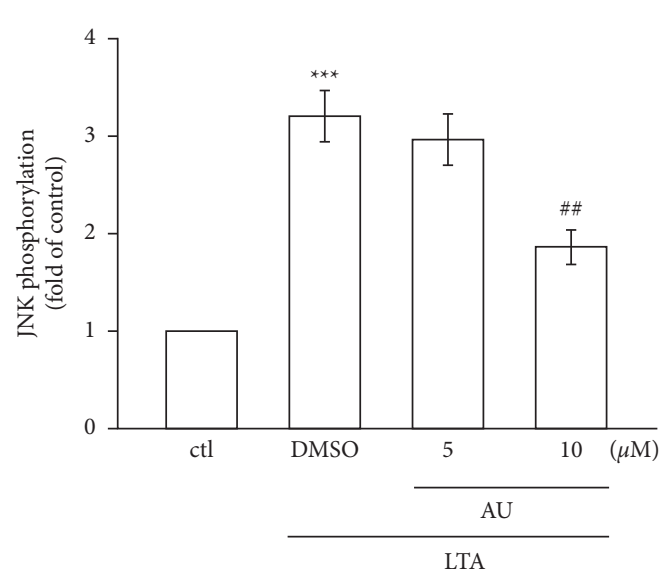

(c)

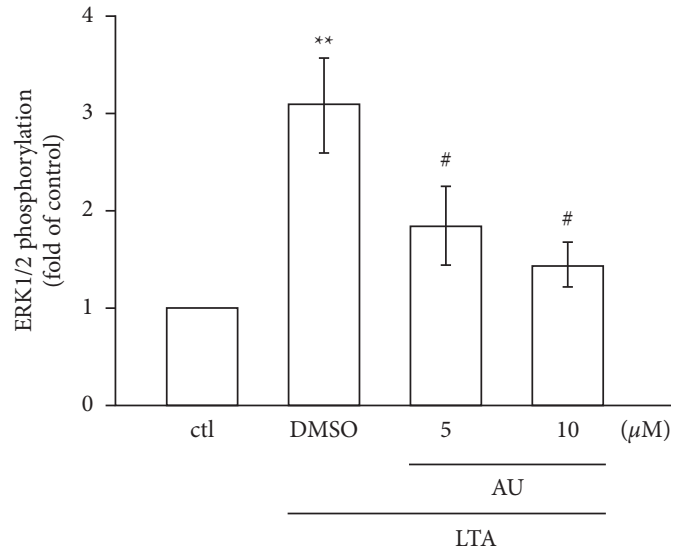

(b)

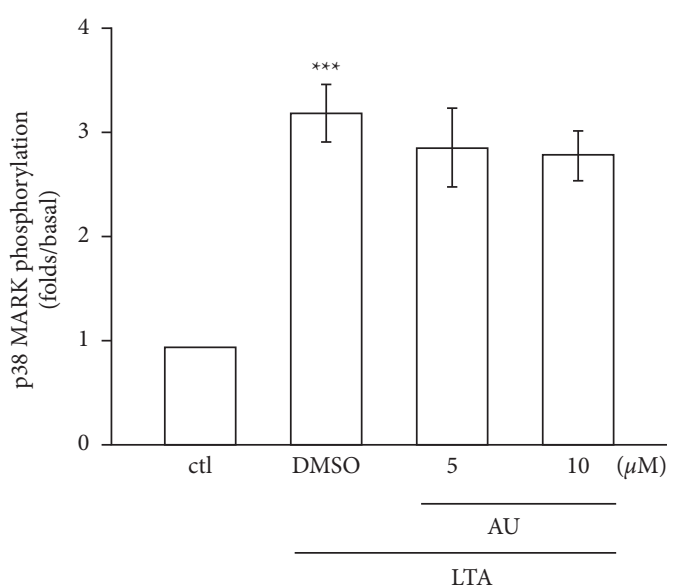

(d)

FIgURE 3: Effects of AU in LTA-induced phosphorylation of MAPKs in RAW 264.7 macrophages. (a) Cells were untreated or pretreated with AU ( 5 and $10 \mu \mathrm{M})$ for $30 \mathrm{~min}$ and were then stimulated with LTA $(5 \mu \mathrm{g} / \mathrm{ml})$ for $1 \mathrm{~h}$. The specific pERK, pJNK, and p38 MAPK antibodies were used to detect these proteins. $\alpha$-Tubulin was used as the internal control. (b)-(d) The statistical values shown are the means \pm S.E.M. of four independent experiments. ${ }^{* *} P<0.01$ and ${ }^{* * *} P<0.001$ vs. the control cells; ${ }^{\#} P<0.05$ and ${ }^{\# \#} P<0.01$ vs. LTA-stimulated cells.

specified that AU at $10-90 \mu \mathrm{M}$ reduced the levels of IL-6 and TNF- $\alpha$ in phytohemagglutinin- (PHA-) stimulated human lymphocytes [30]. A previous study from these authors has also established that AU alleviates IL-4, IL-10, and interferon (IFN- $\gamma$ ) levels [29]. NO plays a role in the pathogenesis of several inflammatory disorders, and its production in activated macrophages via the rate-limiting enzyme iNOS induces several acute and chronic inflammatory conditions [31]. COX-2 is reported to be overexpressed during the course of LPS-induced inflammatory reaction [32]. Studies have described that the overexpression of iNOS and COX-2 stimulates the activation of NO and $\mathrm{PGE}_{2}$ in activated macrophages, respectively. Overproduction of such inflammatory mediators can result in chronic inflammatory diseases [33]. Here, we found that $\mathrm{AU}$ expressively and without causing cytotoxicity inhibits the level of NO in LTA-stimulated RAW 264.7 cells. The AU's inhibitory effect on LTA-induced NO production appears to involve the reduction of iNOS expression. Moreover, AU dramatically inhibited the LTA-induced expression of iNOS, COX-2, TNF- $\alpha$, and IL- $1 \beta$. Okuyama et al. [34] showed that AU suppressed the LPS-induced expression of COX-2, IL$1 \beta$, and TNF- $\alpha$ in astrocytes isolated from the cerebral cortex of ICR mice. Niu et al. found an inhibitory mechanism for AU via IL-2, IFN- $\gamma$, and IL-4 in lymphocytes isolated from C57BL/6 mice [28]. These results are consistent with our results and evident of the anti-inflammatory properties of AU.

The induction of inflammatory mediators involves the activation of multiple signal transduction pathways, 


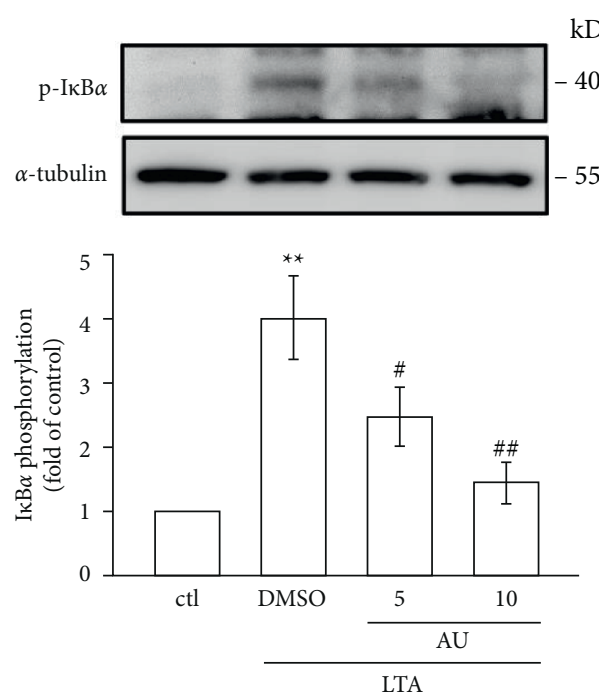

(a)
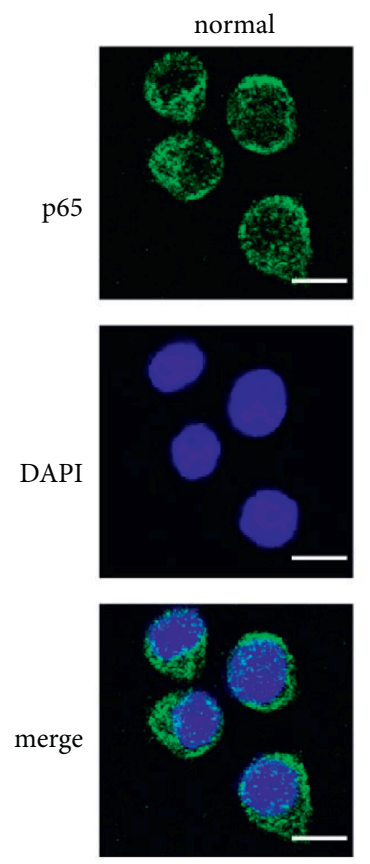

$\mathrm{kDa}$
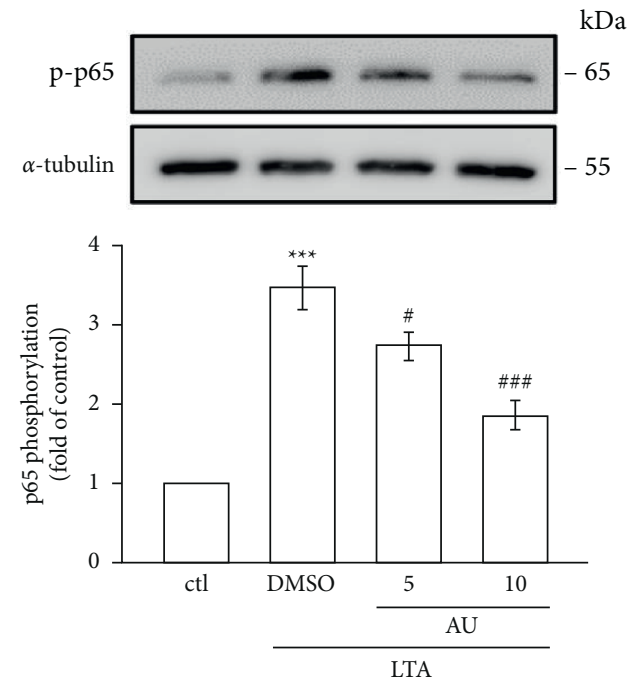

(b)
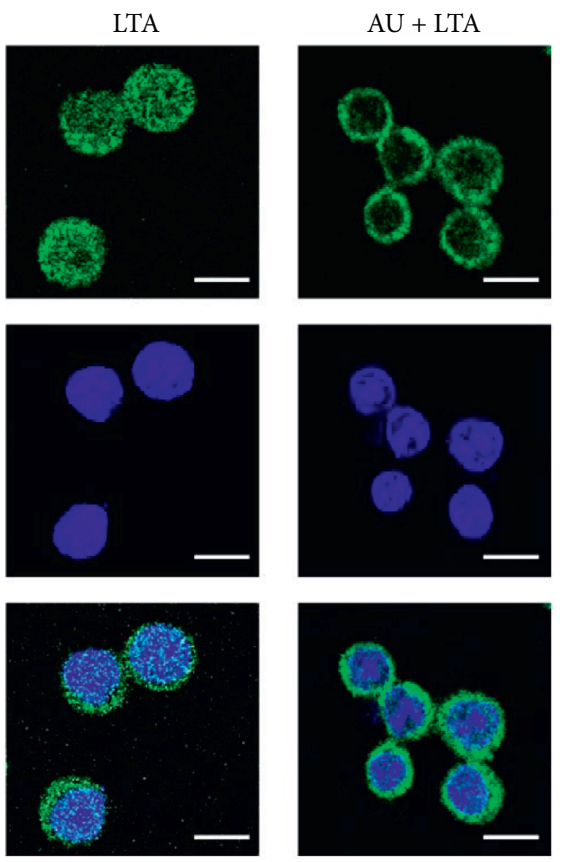

(c)

FIGURE 4: AU controls the NF- $\kappa$ B signaling pathway induced by LTA in RAW 264.7 macrophages. Cells were pretreated with AU (5 and $10 \mu \mathrm{M})$ for $30 \mathrm{~min}$ and were then stimulated with LTA $(5 \mu \mathrm{g} / \mathrm{ml})$ for $1 \mathrm{~h}$. The phosphorylation of (a) I $\kappa \mathrm{B} \alpha$ and (b) p65 in LTA-induced RAW cells was detected as described in Section 2. (c) PTE inhibited LTA-induced p65 nuclear translocation. The values shown are the means \pm S.E.M. of four independent experiments. ${ }^{* *}<0.01$ and ${ }^{* * *}<0.001$ vs. the control cells; ${ }^{\#} P<0.05,{ }^{\# \#} P<0.01$, and ${ }^{\# \# \#} P<0.001$ vs. LTA-stimulated cells.

including mitogen-activated protein kinases (MAPKs) such as p38, ERK, and JNK [35]. It is reported that blocking p38, ERK, and JNK MAPK pathways could decrease iNOS and COX-2 expression and TNF- $\alpha$ and IL- $1 \beta$ production in macrophage inflammation [36]. The MAPK/NF- $\kappa \mathrm{B}$ signaling pathway was conveyed to play a vital role in the expression of TNF- $\alpha$, IL- 6 , IL- $1 \beta$, and COX- 2 in many cell types [37]. Therefore, we examined the effect of AU on MAPK/NF- $\kappa$ B pathway activation. Niu et al. found esculin significantly inhibited the activation of the MAPK pathway in LPS-induced peritoneal macrophages [38]. Guo et al. found both degradation and phosphorylation of $\mathrm{I} \kappa \mathrm{B} \alpha$ and activation of NF- $\kappa \mathrm{B}$ p 65 stimulated by LPS are significantly controlled by imperatorin in RAW 264.7 macrophages [39]. Our recent study found that pterostilbene, a natural substance of blueberry and an analog of resveratrol, significantly inhibited the NF- $\kappa$ B signaling pathway and ERK phosphorylation in RAW 264.7 cells [40]. Thus, it is proposed that coumarin derivatives may inhibit the MAPK/NF- $\kappa \mathrm{B}$ signaling pathway in LPS-induced inflammatory reaction. 


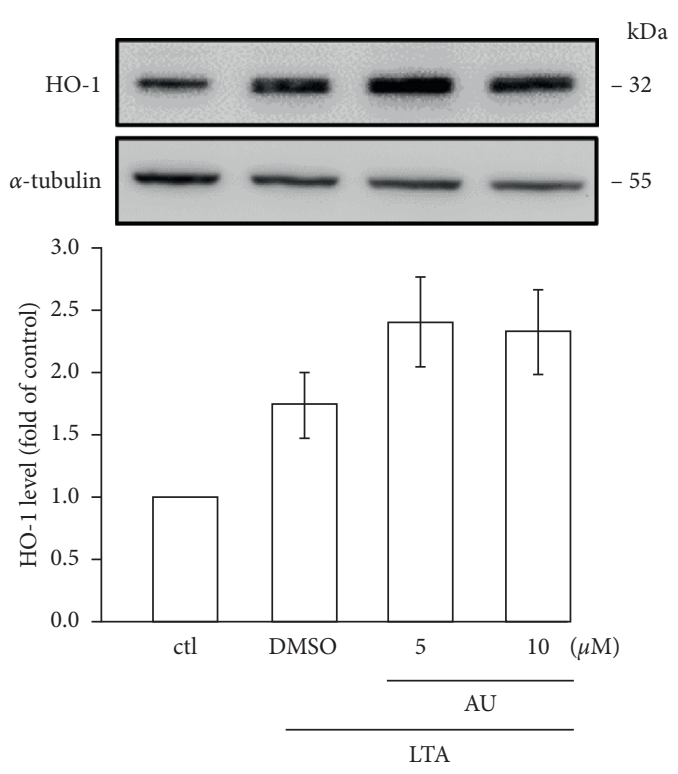

(a)

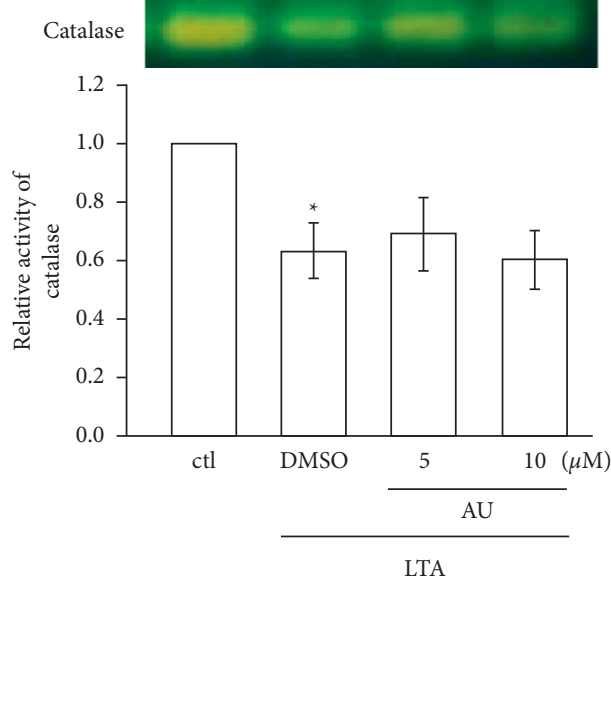

(b)

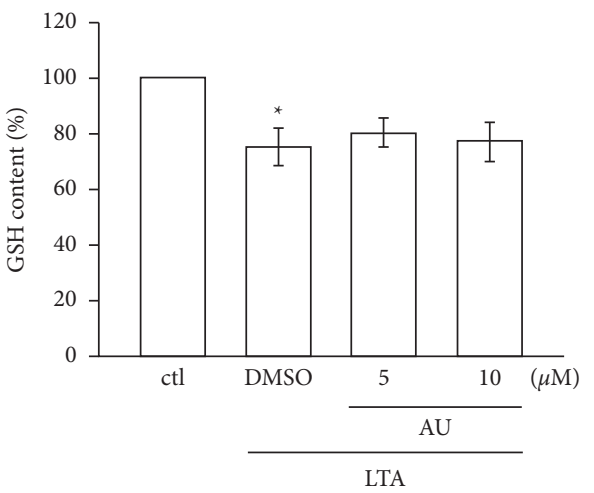

(c)

FIGURE 5: AU enhances antioxidant defense molecules in LTA-stimulated RAW cells. Cells were untreated or pretreated with AU (5 and $10 \mu \mathrm{M})$ for $30 \mathrm{~min}$ followed by LTA $(5 \mu \mathrm{g} / \mathrm{ml})$ for $24 \mathrm{~h}$. The expression of HO-1 (a), catalase (CAT) activity (b), and glutathione (GSH) content (c) in LTA-induced RAW cells was determined using western blotting, native polyacrylamide gel electrophoresis (NATIVE-PAGE), and spectrophotometric analyses, respectively. The values shown are the means \pm S.E.M. of four independent experiments. ${ }^{*} P<0.05$ vs. the control cells.

The results of this study consistently showed that $\mathrm{AU}$ strongly reversed the LTA-induced phosphorylation of JNK and ERK and the nuclear translocation of the p65 subunit. The induction of NF- $\kappa \mathrm{B}$ is controlled by $\mathrm{I} \kappa \mathrm{B}$ kinase (IKK) complex activation, and IKK phosphorylates $\mathrm{I} \kappa \mathrm{B} \alpha$ and initiates ubiquitin-dependent I $\kappa \mathrm{B} \alpha$ degradation [41]. This process could lead NF- $\kappa \mathrm{B}$ translocation to the nucleus, where it attaches to the promoter regions of the target gene and brings proinflammatory mediators such as iNOS, COX2 , TNF- $\alpha$, and IL-6 [42]. The phosphorylation of I $\kappa$ B and p65 can be induced by LTA, and it also can induce p65 translocation from the cytoplasm to nuclei [13]. LTA binds with toll-like receptor (TLR2), which in turn activates NF- $\kappa$ B and consequently translocated to nuclei from the cytoplasm [43]. Hence, these outcomes may propose that AU decreases LTA-induced inflammatory events in RAW cells via inhibiting the activation of JNK/ERK and NF- $\kappa$ B pathways.
Activated oxygen $\left(\mathrm{O}_{2}{ }^{*}\right)$ radicals are metabolized to $\mathrm{H}_{2} \mathrm{O}$ and successively converted to $\mathrm{H}_{2} \mathrm{O}_{2}$ by superoxide dismutase enzymes (SOD) and then to $\mathrm{H}_{2} \mathrm{O}$ by glutathione peroxidase or to $\mathrm{H}_{2} \mathrm{O}_{2}$ and $\mathrm{O}_{2}$ by catalases (CAT) [44]. A previous study found that irisin, a molecule secreted from skeletal muscle in response to physical exercise, plays a regulatory role in an immune system activity and can protect the cell from freeradical-induced cellular oxidative damage by the activation of antioxidative mechanisms [45]. Furthermore, a rise in HO-1 expression was identified to exert both antioxidant and anti-inflammatory effects [44]. HO-1 plays an important role in the protection of oxidative stress in chronic disease [46]. Furthermore, HO-1 has been reported to inhibit various inflammatory responses to exhibit its cellular protective role. Several antioxidants can induce HO-1 expression to cope oxidative damage, and thus, compounds that can activate HO-1 expression may be favorable in the 
treatment of oxidative damage. A natural anti-inflammatory compound curcumin was found to increase the activity of CAT to protect RAW cells from LPS-induced ROS damages [47]. Reduction of reduced glutation (GSH) had reported to lead the progress of several diseases, as GSH inhibits oxidative stress-induced cell damage [48]. Therefore, we examined whether $\mathrm{AU}$ can involve the downstream mechanism via interaction with $\mathrm{HO}-1$ to its antioxidative action. However, AU did not augment HO-1, CAT, and GSH, which postulates that antioxidant mechanisms may not associate to AU's anti-inflammatory role in LTA-induced RAW cells.

\section{Conclusions}

This study shows the anti-inflammatory effects of auraptene via diminishing iNOS, COX-2, IL- $1 \beta$, and TNF- $\alpha$ expression in LTA-induced RAW 264.7 macrophages. The inhibitory property of $\mathrm{AU}$ is mediating at least in part via inhibiting NF- $\kappa$ B, along with the MAPK (JNK and ERK) pathway. Moreover, this study also found that AU's anti-inflammatory role was not depending on antioxidant mechanisms, as AU was not effective in HO-1, CAT, and GSH in the LTAinduced inflammatory RAW 264.7 cells.

\section{Data Availability}

Data can be obtained from the corresponding author on reasonable request.

\section{Conflicts of Interest}

The authors declare no conflicts of interest.

\section{Authors' Contributions}

Chih-Hsuan Hsia, Thanasekaran Jayakumar, Wan-Jung Lu, and Joen-Rong Sheu authors are contributed equally in this work. $\mathrm{CHH}$, TJ, and JRS designed work and wrote the paper. WJL, CWH, and $\mathrm{CHH}$ carried out the experiments. $\mathrm{CH}$, PSB, and WJL performed data analyses. WCH, MM, and YC provided interpretation. All authors approved for the final submission.

\section{Acknowledgments}

This study was funded by grants from the Ministry of Science and Technology of Taiwan (MOST 107-2320-B-038-035MY2 and MOST108-2320-B-038-031-MY3), Taipei Medical University (DP2-107-21121-N-02), Shin Kong Wu Ho-Su Memorial Hospital-Taipei Medical University (SKH-TMU107-04), and Shin Kong Wu Ho-Su Memorial Hospital (2019SKHADR032 and 2021SKHAND005).

\section{References}

[1] P. P. Tak and G. S. Firestein, "NF- $\kappa$ B: a key role in inflammatory diseases," Journal of Clinical Investigation, vol. 107, no. 1, pp. 7-11, 2001.

[2] V. R. Askari, V. Baradaran Rahimi, S. A. Tabatabaee, and R. Shafiee-Nick, "Combination of Imipramine, a sphingomyelinase inhibitor, and $\beta$-caryophyllene improve their therapeutic effects on experimental autoimmune encephalomyelitis (EAE)," International Immunopharmacology, vol. 77, Article ID 105923, 2019.

[3] P. Fu, A. A. Birukova, J. Xing et al., "Amifostine reduces lung vascular permeability via suppression of inflammatory signalling," European Respiratory Journal, vol. 33, no. 3, pp. 612-624, 2009.

[4] V. R. Askari and R. Shafiee-Nick, "The protective effects of $\beta$-caryophyllene on LPS-induced primary microglia M1/M2 imbalance: a mechanistic evaluation," Life Sciences, vol. 219, no. 15 , pp. 40-73, 2019.

[5] C.-j. Huang and M.-C. Wu, "Differential effects of foods traditionally regarded as "heating" and "cooling" on prostaglandin E2 production by a macrophage cell line," Journal of Biomedical Science, vol. 9, no. 6, pp. 596-606, 2002.

[6] A. Murakami, T. Shigemori, and H. Ohigashi, "Zingiberaceous and citrus constituents, $1^{\prime}$-acetoxychavicol acetate, zerumbone, auraptene, and nobiletin, suppress lipopolysaccharide-induced cyclooxygenase-2 expression in RAW264.7 murine macrophages through different modes of action," Journal of Nutrition, vol. 135, no. 12, pp. 2987s-2992s, 2005.

[7] A. Rahal, A. Kumar, V. Singh et al., "Oxidative stress, prooxidants, and antioxidants: the interplay," BioMed Research International, vol. 2014, Article ID 761264, 19 pages, 2014.

[8] V. Baradaran Rahim, M. T. Khammar, H. Rakhshandeh, A. Samzadeh-Kermani, A. Hosseini, and V. R. Askari, "Crocin protects cardiomyocytes against LPS-Induced inflammation," Pharmacological Reports, vol. 71, no. 6, pp. 1228-1234, 2019.

[9] M. Khosravi, A. Poursaleh, G. Ghasempour, S. Farhad, and M. Najafi, "The effects of oxidative stress on the development of atherosclerosis," Biological Chemistry, vol. 400, no. 6, pp. 711-732, 2019.

[10] J. E. Wang, M. K. Dahle, M. McDonald, S. J. Foster, A. O. Aasen, and C. Thiemermann, "Peptidoglycan and lipoteichoic acid in gram-positive bacterial sepsis: receptors, signal transduction, biological effects, and synergism," Shock, vol. 20, no. 5, pp. 402-414, 2003.

[11] J. C. Leemans, M. Heikens, K. P. M. van Kessel, S. Florquin, and T. van der Poll, "Lipoteichoic acid and peptidoglycan from Staphylococcus aureus synergistically induce neutrophil influx into the lungs of mice," Clinical and Vaccine Immunology, vol. 10, no. 5, pp. 950-953, 2003.

[12] E. M. Greenfield, M. A. Beidelschies, J. M. Tatro, V. M. Goldberg, and A. G. Hise, "Bacterial pathogen-associated molecular patterns stimulate biological activity of orthopaedic wear particles by activating cognate Toll-like receptors," Journal of Biological Chemistry, vol. 285, no. 42, pp. 32378-32384, 2010.

[13] H.-C. Chang, K.-H. Lin, Y.-T. Tai, J.-T. Chen, and R.-M. Chen, "Lipoteichoic acid-induced TNF- $\alpha$ and IL- 6 gene expressions and oxidative stress production IN macrophages are suppressed BY ketamine through downregulating toll-like receptor 2-MEDIATED activation OF ERK1/2 and NFkB," Shock, vol. 33, no. 5, pp. 485-492, 2010.

[14] F. Epifano, S. Genovese, and M. Curini, "Auraptene: phytochemical and pharmacological properties," in Phytochemistry Research Progressed, T. Matsumoto, Ed., pp. 145-162, Nova Science Publishers Inc., New York, NY, USA, 2008.

[15] M. Prince, Y. Li, A. Childers, K. Itoh, M. Yamamoto, and H. E. Kleiner, "Comparison of citrus coumarins on carcinogen-detoxifying enzymes in Nrf2 knockout mice," Toxicology Letters, vol. 185, no. 3, pp. 180-186, 2009. 
[16] A. Murakami, Y. Nakamura, and T. Tanaka, "Suppression by citrus auraptene of phorbol ester- and endotoxin-induced inflammatory responses: role of attenuation of leukocyte activation," Carcinogenesis, vol. 21, no. 10, pp. 1843-1850, 2000.

[17] K. Takeda, H. Utsunomiya, S. Kakiuchi et al., "Citrus auraptene reduces Helicobacter pylori colonization of glandular stomach lesions in Mongolian gerbils," Journal of Oleo Science, vol. 56, no. 5, pp. 253-260, 2007.

[18] F. Soltani, F. Mosaffa, M. Iranshahi et al., "Auraptene from Ferula szowitsiana protects human peripheral lymphocytes against oxidative stress," Phytotherapy Research, vol. 24, no. 1, pp. 85-89, 2010.

[19] F. Epifano, G. Molinaro, S. Genovese, R. T. Ngomba, F. Nicoletti, and M. Curini, "Neuroprotective effect of prenyloxycoumarins from edible vegetables," Neuroscience Letters, vol. 443, no. 2, pp. 57-60, 2008.

[20] T. Tanaka, H. Sugiura, and R. Inaba, "Immunomodulatory action of citrus auraptene on macrophage functions and cytokine production of lymphocytes in female BALB/c mice," Carcinogenesis, vol. 20, no. 8, pp. 1471-1476, 1999.

[21] T. Tanaka, H. Kohno, and M. Murakami, "Suppressing effects of dietary supplementation of the organoselenium 1,4henylenebis(methylene)selenocyanate and the Citrus antioxidant auraptene on lung metastasis of melanoma cells in mice," Cancer Research, vol. 14, pp. 3713-3716, 2000.

[22] V. D. La, L. Zhao, F. Epifano, S. Genovese, and D. Grenier, "Anti-inflammatory and wound healing potential of citrus auraptene," Journal of Medicinal Food, vol. 16, no. 10, pp. 961-964, 2013.

[23] W. Woodbury, A. K. Stahmann, and M. A. Stahman, “An improved procedure using ferricyanide for detecting catalase isozymes," Analytical Biochemistry, vol. 44, no. 1, pp. 301-305, 1971.

[24] J. N. Sharma, A. Al-Omran, and S. S. Parvathy, "Role of nitric oxide in inflammatory diseases," Inflammopharmacology, vol. 15, no. 6, pp. 252-259, 2007.

[25] X. Li and G. R. Stark, "NF- $\kappa$ B-dependent signaling pathways," Experimental Hematology, vol. 30, no. 4, pp. 285-296, 2002.

[26] A. Singh, R. Kukreti, L. Saso, and S. Kukreti, "Oxidative stress: a key modulator in neurodegenerative diseases," Molecules, vol. 24, no. 8, p. 1583, 2019.

[27] S. Genovese and F. Epifano, "Auraptene: a natural biologically active compound with multiple targets," Current Drug Targets, vol. 12, no. 3, pp. 381-386, 2011.

[28] X. Niu, Z. Huang, L. Zhang, X. Ren, and J. Wang, "Auraptene has the inhibitory property on murine $\mathrm{T}$ lymphocyte activation," European Journal of Pharmacology, vol. 750, pp. 8-13, 2015.

[29] V. R. Askari, V. Baradaran Rahimi, S. A. Rezaee, and M. H. Boskabady, "Auraptene regulates Th 1/Th 2/T Reg balances, NF- $\kappa \mathrm{B}$ nuclear localization and nitric oxide production in normal and Th 2 provoked situations in human isolated lymphocytes," Phytomedicine, vol. 43, pp. 1-10, 2018.

[30] V. R. Askari, V. B. Rahimi, and R. Zargarani, "Anti-oxidant and anti-inflammatory effects of auraptene on phytohemagglutinin (PHA)-induced inflammation in human lymphocytes," Pharmacological Reports, vol. 73, pp. 54-162, 2021.

[31] F. Aktan, "iNOS-mediated nitric oxide production and its regulation," Life Sciences, vol. 75, no. 6, pp. 639-653, 2004.

[32] J. K. Lee, B. C. Sayers, K.-S. Chun et al., "Multi-walled carbon nanotubes induce COX-2 and iNOS expression via MAP kinase-dependent and -independent mechanisms in mouse
RAW264.7 macrophages," Particle and Fibre Toxicology, vol. 9, no. 1, p. 14, 2012.

[33] F. Zhao, L. Chen, C. Bi, M. Zhang, W. Jiao, and X. Yao, "In vitro anti-inflammatory effect of picrasmalignan $\mathrm{A}$ by the inhibition of iNOS and COX-2 expression in LPS-activated macrophage RAW 264.7 cells," Molecular Medicine Reports, vol. 8, no. 5, pp. 1575-1579, 2013.

[34] S. Okuyama, M. Morita, M. Kaji et al., "Auraptene acts as an anti-inflammatory agent in the mouse brain," Molecules, vol. 20, no. 11, pp. 20230-20239, 2015.

[35] S. H. Kim, C. J. Smith, and L. J. Van Eldik, "Importance of MAPK pathways for microglial pro-inflammatory cytokine IL-1 $\beta$ production," Neurobiology of Aging, vol. 25, no. 4, pp. 431-439, 2004.

[36] G. Pearson, F. Robinson, T. Beers Gibson et al., "Mitogenactivated protein (MAP) kinase pathways: regulation and physiological functions," Endocrine Reviews, vol. 22, no. 2, pp. 153-183, 2001.

[37] O. A. Olajide, M. A. Aderogba, and B. L. Fiebich, "Mechanisms of anti-inflammatory property of Anacardium occidentale stem bark: inhibition of NF- $\kappa$ B and MAPK signalling in the microglia," Journal of Ethnopharmacology, vol. 145, no. 1, pp. 42-49, 2013.

[38] X. Niu, Y. Wang, W. Li et al., "Esculin exhibited anti-inflammatory activities in vivo and regulated TNF- $\alpha$ and IL- 6 production in LPS-stimulated mouse peritoneal macrophages in vitro through MAPK pathway," International Immunopharmacology, vol. 29, no. 2, pp. 779-786, 2015.

[39] W. Guo, J. Sun, L. Jiang et al., "Imperatorin attenuates LPSinduced inflammation by suppressing NF- $\kappa \mathrm{B}$ and MAPKs activation in RAW 264.7 macrophages," Inflammation, vol. 35, no. 6, pp. 1764-1772, 2012.

[40] T. Jayakumar, M. P. Wu, and J. R. Sheu, "Involvement of antioxidant defenses and NF- $\kappa \mathrm{B} / \mathrm{ERK}$ signaling in anti-inflammatory effects of pterostilbene, a natural analogue of resveratrol," Applied Sciences, vol. 11, pp. 1-12, 2021.

[41] A. Oeckinghaus and S. Ghosh, "The NF-kappaB family of transcription factors and its regulation," Cold Spring Harbor Perspectives in Biology, vol. 1, no. 4, Article ID a000034, 2009.

[42] T. Liu, L. Zhang, D. Joo, and S. C. Sun, "NF- $\kappa$ B signaling in inflammation," Signal transduction and targeted therapy, vol. 2, Article ID 17023, 2017.

[43] C.-Y. Chuang, T.-L. Chen, and R.-M. Chen, "Molecular mechanisms of lipopolysaccharide-caused induction of surfactant protein-A gene expression in human alveolar epithelial A549 cells," Toxicology Letters, vol. 191, no. 2-3, pp. 132-139, 2009.

[44] B. Usluoğullari, C. A. Usluogullari, F. Balkan, and M. Orkmez, "Role of serum levels of irisin and oxidative stress markers in pregnant women with and without gestational diabetes," Gynecological Endocrinology: The Official Journal of the International Society of Gynecological Endocrinology, vol. 33, no. 5, pp. 405-407, 2017.

[45] A. I. Mazur-Bialy, K. Kozlowska, and E. Pochec, "Myokine irisin-induced protection against oxidative stress in vitro. Involvement of heme oxygenase- 1 and antioxidazing enzymes superoxide dismutase-2 and glutathione peroxidase," Journal of Physiology and Pharmacology, vol. 69, no. 1, pp. 117-125, 2018.

[46] B. G. Park, C. I. Yoo, H. T. Kim, C. H. Kwon, and Y. K. Kim, "Role of mitogen-activated protein kinases in hydrogen peroxide-induced cell death in osteoblastic cells," Toxicology, vol. 215, no. 1-2, pp. 115-125, 2005. 
[47] X. Lin, D. Bai, Z. Wei et al., "Curcumin attenuates oxidative stress in RAW264.7 cells by increasing the activity of antioxidant enzymes and activating the Nrf2-Keap1 pathway," PLoS One, vol. 14, no. 5, Article ID e0216711, 2019.

[48] M. Deponte, "The incomplete glutathione puzzle: just guessing at numbers and figures?" Antioxidants and Redox Signaling, vol. 27, no. 15, pp. 1130-1161, 2017. 